\title{
Dispersive Motions in the $F$-Region of the Equatorial Ionosphere
}

\author{
D.K. BAMGBOYE \\ Physics Department, University of Ibadan, Ibadan, Nigeria*
}

(Received February 12, 1973)

\begin{abstract}
Some results of "dispersion" analysis of $F$-region drift data obtained at Ibadan (dip $\left.6^{\circ} \mathrm{S}\right)$ are presented. On a number of occasions marked dispersion involving a velocity variation of about $60 \mathrm{msec}^{-1}$ or more from low to high fading frequencies has been found. The results suggest that in the "correlation" analysis the "apparent" velocity probably measures the motion better than the "true" velocity, whether or not dispersion is present. It is also found that the motions occurring at this latitude are not always in the east-west direction. Occasionally, two independent motions corresponding to different fading frequency ranges and moving at right angles to each other may be involved.
\end{abstract}

\section{Introduction}

The spaced receiver ionospheric drift measurements by the conventional analysis methods of "similar fades" introduced by Mitra (1949) and of "full correlation" by Briggs et al. (1950) and Phillips and Spencer (1955) have been made for several years at a number of ionospheric stations round the world. But although the broad world-wide pattern of the velocity of the radio diffraction pattern formed on the ground is now fairly well known, as shown by KENT and Wright (1968) and KENT (1970), the problem of interpreting the observed motion in ionospheric terms remains largely unsolved. For instance, it is not certain whether what is observed is a pure bodily drift of ionization irregularities or merely a progression of some form of atmospheric waves through the ionosphere, or both. In either of these cases it is of interest to know whether the irregularities (or Fourier components of the wave perturbation) all move with same average speed and in the same average direction as is basically assumed in the above conventional methods of analysis.

\section{Dispersion Analysis}

In recent years a number of workers have carried out spectral analysis of the three receiver fading records in order to test the motion for dispersion as

* Part of this work was carried out while the author was on study leave at the University College of Wales, Aberystwyth, U.K. 
first suggested by JONES and MAUDE (1965). The basic assumption of the "dispersion" method is that the ground diffraction pattern consists of a number of plane waves, each with a unique frequency, all moving over the receiving array. Such a diffraction pattern could be produced if ionospheric irregularities or Fourier components of an ionospheric wave perturbation move with different velocities.

In the "dispersion" method of Briggs and Golley (1968) and Jones and MAUde (1968) based on the work by Goodman et al. (1961) auto- and crosscorrelation functions are obtained from amplitude samples as in the "full correlation" method. These functions are then Fourier transformed to give auto- and cross-spectral functions at a number of frequencies. These spectra are useful in estimating at what range of frequencies there is significant signal amplitude present above the noise level of the records; the auto-spectrum is often referred to as the "power spectrum" and the modulus of the cross-spectrum as the "crosspower." The phases of the cross-spectra of record pairs are converted into time shifts and from these and the receiver spacing the speed and direction of the motion of each frequency component are deduced. Any variation of velocity with frequency is called "dispersion."

Results of dispersion analysis so far published have been mainly from highlatitude stations and on the $D$ - and $E$-region by reflection by Gossard (1967), Haug and Pettersen (1970), Golley and Rossiter (1971) and Jones and MAUde (1972), and on the whole ionosphere by transmission from radio star and satellite sources by Briggs and Golley (1968) and PAPAGIANNis and Elkins (1970). Important results have been obtained some of which are mentioned briefly later. Data on the $F$-region observed by reflection have, however, not been so treated. It is also doubtful if any drift data on the low-latitude ionosphere have so far been tested for dispersion.

Ibadan is one of the few low-latitude stations that have made drift measurements for several years using the conventional methods. Because the results of the dispersion analysis is likely to affect the future of drift measurements generally, the data for this station is now being subjected to this analysis in order to assess previous results. This paper is a report on the $F$-region.

The $F$-region was observed by reflection of pulsed radio waves of frequencies $5.7 \mathrm{MHz}$ by day and $2.4 \mathrm{MHz}$ by night. The fading records were obtained at the vertices of a right-angled isosceles triangle of short sides $127 \mathrm{~m}$ oriented with the hypotenuse lying north-south. In this work 360 amplitude samples at a regular sampling interval of 0.2 second have been most frequently used. With slow fading records which sometimes occur, however, sampling intervals of 0.4 and 0.8 second have been used. Velocities quoted have been divided by a factor of 2 to make them refer to the ionospheric diffracting screen. 


\section{Results}

Illustrative examples of the results obtained are presented in Figs. 1-4. Fading frequencies of daytime records are usually higher than those of the night time, but no differences have been observed in the pattern of behaviour of the velocities for the day and the night data. For this reason the examples given have been freely drawn from all the data without separating the night from the day. The time of observation has however been indicated in each case.

\subsection{Spectral power}

Three typical examples of the spectral power obtained in this analysis are shown in Fig. 1 where in each case the cross power of the three record pairs have been plotted as functions of frequency. The amplitudes of the cross spectra are complex and what have been plotted here are their moduli. The power spectra (not shown) are similar to the cross power but, in general, they fall off with frequency more gradually than the cross power. This is to be expected since although the three fading records from the spaced receivers are similar they are not identical. For the same reason it can be seen from Fig. 1 that in each example the three cross powers show some differences which may be quite

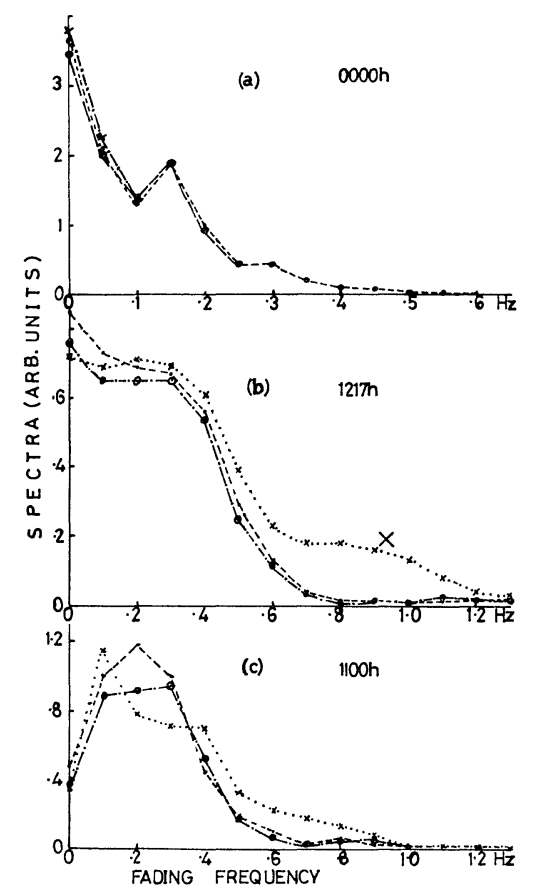

Fig. 1. Typical spectra of fading records. The moduli of the amplitudes of the three cross spectra between record pairs are plotted against frequency in each of the three examples. 
small as in Fig. 1(a) or appreciable as Fig. 1(b) and (c). This is similar to what one observes in correlation functions.

The "cut off" frequency of the spectra varies from one observation to another being about $0.35,0.7$ and $0.6 \mathrm{~Hz}$ in the examples of Fig. 1(a), (b) and (c), respectively. It is sometimes lower than these values, but cases in which it is higher than about $0.8 \mathrm{~Hz}$ are not common. Because the three cross spectra in each observation are not identical, it is important when using them to examine over what range of frequencies there is significant power not to pick any one cross spectrum arbitrarily. Figure 1(a) and (b) will illustrate this point. While an arbitrary choice of a representative power spectrum would be satisfactory in Fig. 1(a), it could lead to errors in Fig. 1(b) if the cross spectrum marked X happened to be the one picked. For although the latter has appreciable power likely to be above noise level up to about $1.0 \mathrm{~Hz}$, the other two indicate that there is probably no significant power common to all the three receiving points above about $0.65 \mathrm{~Hz}$. A more reasonable thing to do would seem to be to adopt the mean or, better still, the cross spectrum with the lowest "cut off" frequency as providing the best guide on the coherent frequencies present in all the three fading records. In a similar way the cross power between two records is a better guide to coherent frequencies present than the power spectrum of an individual record because the former has the lower "cut off" frequency. Furthermore, for coherency at any frequency, the phases of the three cross spectra must satisfy the relation

$$
P_{13}=P_{12}+P_{23}
$$

where $1,2,3$ refer to the three receiving points, $P_{12}$ is the phase lag between points 1 and 2, and similarly for $P_{13}$ and $P_{23}$. These criteria have been adhered to in the present analysis.

\subsection{Velocities}

The frequency dependence of velocities obtained are illustrated by the examples of Figs. 2-4. Throughout these examples each Fourier velocity is shown as a dot carrying an arrow pointing towards the direction of motion of that particular frequency, magnetic north being taken as parallel to the velocity axis. The corresponding "apparent" velocity $V_{\mathrm{a}}$ and "true" velocity $V$ as well as their directions $\phi_{\mathrm{a}}$ and $\phi$ from the correlation analysis are shown in a similar manner to the right side, while the magnitude of the characteristic random velocity $V_{\mathrm{c}}$ is indicated by a line parallel to the velocity axis. The representative cross power for each example is also shown.

3.2.1 Constant velocity: Figure 2 gives three examples in which the velocity is, on the average, independent of frequency. In each case the direction of motion is very closely the same at all frequencies and the small variations in the speed can be attributed to the errors of the analysis or random changes 


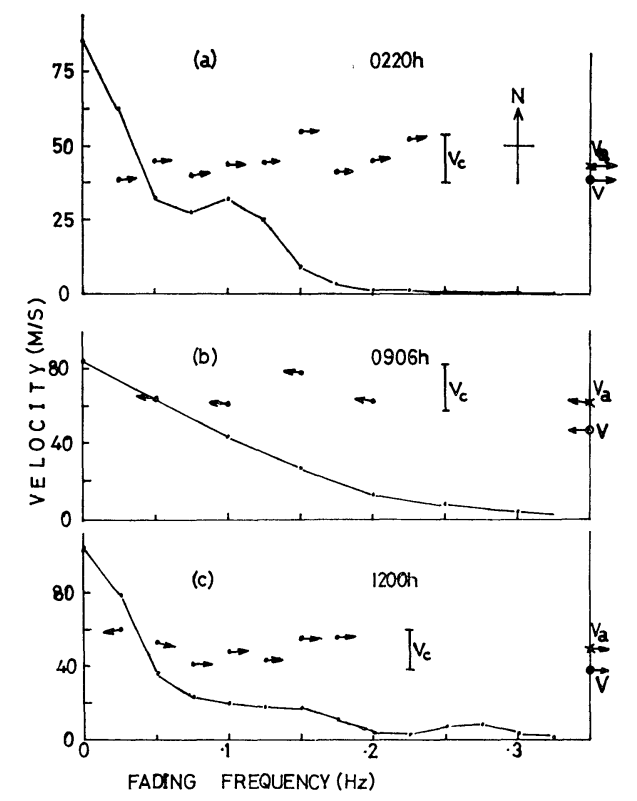

Fig. 2. Velocity independent of frequency. The corresponding apparent velocity $V_{\mathrm{a}}$, the "true" velocity $V$ and the random velocity $V_{\mathrm{c}}$ are shown to the right. The curve gives the representative cross power in each case.

present. In each case also the "apparent" velocity $V_{\mathrm{a}}$ agrees with the mean of the Fourier velocities, while the "true" velocity falls below even the lowest Fourier velocity. Furthermore, the characteristic random velocity $V_{\mathrm{c}}$ corresponds very well to the spread of the Fourier velocities.

3.2.2 Positive dispersion: Examples of observations in which velocity is found to increase with increasing frequency are shown in Fig. 3. The velocity increases by about $80 \mathrm{msec}^{-1}$ over the fading frequency range of coherent power in Fig. 3(a) and (c) and about $60 \mathrm{msec}^{-1}$ in Fig. 3(b). In each example the direction of motion is consistent at all frequencies, being westward in (a), southward in (b) and an average of about $65^{\circ}$ west of the south in (c). The variations of velocity with frequency are approximately linear, but it is in case (c) only that the linearity is through the origin. However, some of the observations gave positive dispersions which are either non-linear or linear only over a portion of the spectrum.

"Apparent" velocities can be seen to correspond to velocities at or near the middle frequency of the fading spectrum while the "true" velocities correspond more or less to the lower limit of the Fourier velocities in the examples of Fig. $3(a)$ and (b). This is what is commonly observed when dispersion is present. It should be noted also that in these cases the random velocity $V_{\mathrm{c}}$ is somewhat smaller than the range of Fourier velocities involved, suggesting that $V_{\mathrm{c}}$ is per- 


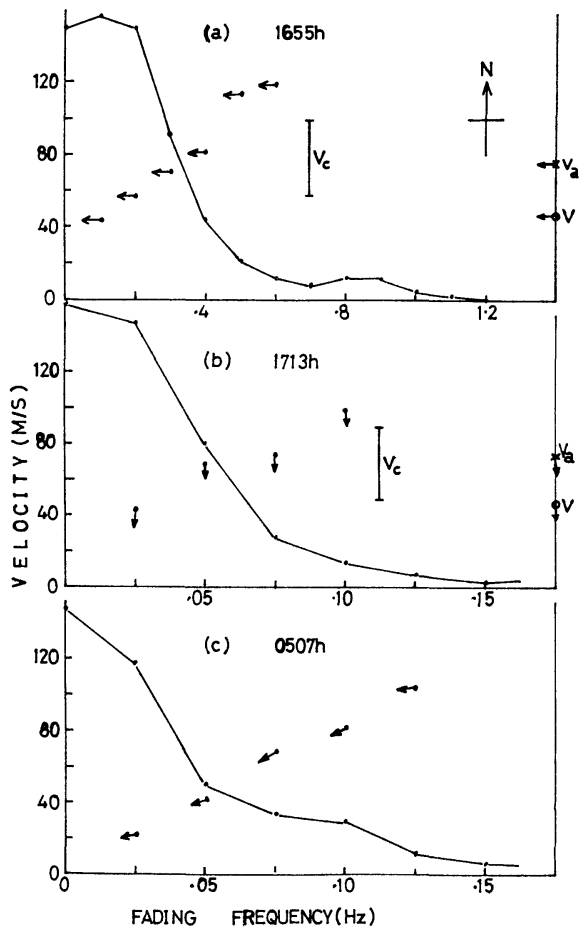

Fig. 3. Positive dispersion, and the corresponding correlation analysis results in cases (a) and (b). The curve gives the representative cross-power in each case.

haps not simply related to the spread of component velocities in these cases. Correlation analysis results are not shown in Fig. 3(c) because this example gave abnormally large $V_{\mathrm{a}}$ and an imaginary $V_{\mathrm{c}}$ so that the correlation analysis broke down. The very systematic velocities obtained at different fading frequencies for the same example may well be one of the merits of the "dispersion" analysis.

3.2.3 Other behaviour: With a number of other observations the velocityfrequency graphs behave in various other ways some of which are illustrated by the examples of Fig. 4. In Fig. 4(a) the low frequencies show a tendency to move westward while the higher frequencies are moving southward. This example is particularly striking because the spectral power appears to be significant only over the two different ranges of frequency approximately corresponding to the two velocity groups. As shown, the "apparent" velocity in this case corresponds to the velocity of the mid frequency of the southward motion, both in magnitude and direction, but here again $V_{\mathrm{c}}$ was imaginary.

A few results show negative dispersion as in Fig. 4(b) where the range of velocity is about $-70 \mathrm{msec}^{-1}$. Here the "apparent" velocity again corresponds to the velocity of the mid frequency range just as in the case of positive dispersion; 


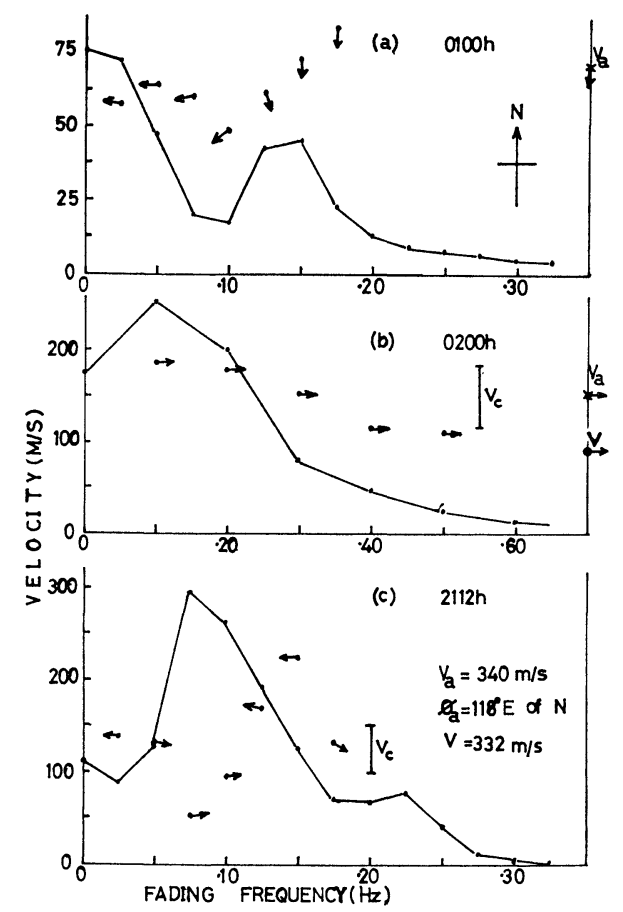

Fig. 4. Examples of other types of dispersion observed.

the "true" velocity also falls below the smallest Fourier velocity, but the random velocity $V_{\mathrm{c}}$ is about the same as the range of the velocities.

Some other results show little consistency either in speed or direction and an example is given in Fig. 4(c). Even where the directions are consistent, as in some cases, the speeds show large random variations with frequency. The randomness is sometimes reflected in the correlation analysis as in the example of Fig. 4(c) where a large "apparent" velocity of $340 \mathrm{msec}^{-1}$ in a direction $120^{\circ}$ east of the north and a comparatively small characteristic random velocity of $50 \mathrm{msec}^{-1}$ were obtained.

\section{Discussion}

This report covers the results of about $35 \mathrm{~F}$-region records so far analysed and well over a third of these show definite positive dispersion. Whether or not this proportion is representative of the Ibadan $F$-region data will subsequently be determined when a greater number of records has been analysed. Cases of negative dispersion were very few, but in general the results do show that at Ibadan the assumption that all Fourier components of the ground diffraction of $F$-region reflected signals move with the same average velocity, as used in previous treatment of data for this station, is only sometimes valid. Even where 
the assumption is found to be valid, these results of dispersion analysis are not without important implications for the conventional analysis. It is usual in the "correlation" method to regard the "apparent" velocity, $V_{\mathrm{a}}$, as trivial and only a guide to the "true" velocity, $V$. The two are related through a characteristic random velocity, $V_{\mathrm{c}}$, by

$$
V_{\mathrm{a}} / V=1+\left(V_{\mathrm{c}} / V\right)^{2} \text {. }
$$

In the examples of Fig. 2 the small variations of speed (not direction) with frequency are random about the mean and $V_{\mathrm{c}}$ in each case is just of the right order to account for them. It may be concluded that there is no evidence of dispersion in such cases. For them, therefore, the "correlation" analysis seems adequate. However, the "apparent" velocity $V_{\mathrm{a}}$ (which agrees with the mean Fourier velocity) and not the "true" velocity (which is smaller than the smallest Fourier velocity) should be adopted. The adoption of the under estimated "true" velocity may well be partly responsible for the fact that the observed east-west drift velocity in the low latitude areas, while agreeing reasonably well with the prediction of the electrodynamic theory as shown in the review by Kent (1970, his Fig. 15), is in general lower than the prediction.

Using an array of 89 aerials Golley and Rossiter (1970) found that "apparent" velocities obtained in the correlation analysis were independent of the size of the triangular array used. The "true" velocity on the other hand increased with triangle size until it agreed with the velocity obtained from spatial correlation analysis (which they assumed was the correct value) when an optimum triangle size of sides estimated at about $300 \mathrm{~m}$ for the $E$-region was used. The "true" velocity could thus be low if the triangle was smaller than the optimum size. However, although the apparent velocity they obtained tended to be higher than the spatial correlation velocity, it is clear from their Fig. 3 that the two are quite comparable in view of the errors usually involved in these analyses. Thus, even when obtained from triangle arrays of order one or two wavelengths of the probing radio waves as have been commonly done, the apparent velocity is a good estimate of what is presumed to be the correct value to which the "true" velocity tends as triangle size is increased. The very good agreement between apparent velocity and the mean Fourier velocity whether or not dispersion is present as obtained here also suggests that the apparent velocity is a good estimate of whatever is the correct value. It is also useful to note that the above authors in a subsequent work, GolLEy and Rossiter (1971), found that the dispersion analysis was independent of triangle size and that apparent velocity corresponded to velocities in the mid frequency ranges of the spectra when dispersion was present.

According to the present results, the characteristic random velocity $V_{\mathrm{c}}$ provides a good measure of the random variations of the magnitude of the velocity when there is no dispersion or the spread of the velocity when dispersion 
is present, although in the latter case it is unlikely that $V_{\mathrm{c}}$ will account for the large range of Fourier velocities on a one to one correspondence basis.

One further important point to note about these results is that the motion is not always east-west. In some cases the direction is inclined to the east-west direction as in Fig. 3(c) and in some others it is wholly north-south as in Fig. 3 (b). To examine the north-south motion fully at this station a much wider receiver separation than used here is, of course, required, but even these short baseline results again indicate, in agreement with previous work by the author, BAMGBOYE (1971) that north-south motions, whatever their nature, are sometimes present at this latitude.

The example of Fig. 4(a) and similar others suggest that what may sometimes be involved are two independent motions (independent at least in terms of the dimensions and directions of what are moving) at right angles to each other, one east-west and the other north-south. The presence of such two independent motions simultaneously in the $F$-region is not inconceivable. The westward motion might for instance be purely an electrodynamic drift of ionization while the southward motion might be evidence of wave disturbances propagating north-south and imparting the motion of neutral gas to ionization through collisional interaction as suggested by Hooke (1968).

The presence of two directions of motion at the same time could, however, have been due to crossing fringe effects discussed by Felgate and Golley (1971a). These authors showed that with a small number of specular points in the ionosphere interference fringes could appear in the ground pattern. With two such set of fringes in the pattern temporal Fourier analysis could give two velocities, one for each set of fringes.

Several aspects of these results for the $F$-region at Ibadan agree with the results for others stations, although the latter results refer to the $D$-and $E$-region by reflection and the $F$-region by transmission.

At Aberystwyth Jones and Maude $(1965,1968,1972)$ found for the $E$ region that on some occasions all the Fourier components moved, on the average, with the same velocity which was close to the apparent velocities obtained from the "correlation" and "similar fades" analyses. In a number of other cases the velocity varied randomly with frequency while in more than 25 percent of all cases they observed positive dispersion for which the velocities in the mid-frequency range agreed with the apparent velocities of the conventional analyses.

BRIGGS and Golley (1968) using radio star scintillations found that for the $F$-region a majority of their results showed positive dispersion. However, their results indicate a tendency for the apparent velocity to correspond to the velocity of the high frequency end of the spectrum.

Golley and Rossiter (1971) found positive dispersion in about 23 percent of cases of their partial reflection observations of the $D$ and lower $E$-region and 14 percent in the case of data for total reflection from the $E$-region. As has been 
noted, their apparent velocity results corresponded to velocities in the midfrequency ranges of the spectra.

As regards the cause of the observed dispersion, Felgate and Golley (1971b) have shown that substantial changes in the velocity during the period of an observation could produce positive dispersion. No such changes were detected in the records used for the present analysis. Surface gravity waves have been suggested as the causative mechanism in the $D$ and $E$-regions by JoNES and MAUde $(1965,1972)$ while $F$-region dispersion observed by transmission have been explained in terms of vertical variations of velocity by BRIGGS and Golley (1968).

In the case of the $F$-region observation by reflection if, as is likely, there are irregularities of several scales present simultaneously or if dispersive wave motions are present at the level of reflection, these will contribute to the dispersion observed in the ground diffraction pattern. Irregularities at other heights could also possibly contribute.

\section{Conclusions}

It has been shown from experimental results that at the low-latitude station of Ibadan marked positive dispersion is sometimes present in the motion of the ground diffraction pattern of $F$-region reflected radio waves. Both in the dispersive and non-dispersive cases it is the "apparent," and not the "true," velocity from the correlation analysis that correspond to the average velocity of all Fourier components. In cases where no significant dispersion has been observed the characteristic random velocity $V_{\mathrm{c}}$ of the correlation analysis appears to be meaningful as accounting directly for the random changes present in the motion. It appears, however, that $V_{\mathrm{c}}$ will not usually account directly for the large velocity range of order $60-80 \mathrm{msec}^{-1}$ observed when a definite positive dispersion is present.

The results also show that $F$-region motions over Ibadan are not always in the east-west direction. In some cases the motion is north-south or in some other direction. Furthermore, it appears that, on a few occasions, what may be involved are two types of motion giving rise to two ranges of fading frequencies and moving at right angles to each other, one east-west and the other north-south.

This work was carried out partly under a research grant by the University of Ibadan and partly while the author, on study leave at the University College of Wales, Aberystwyth, was supported by a Commonwealth Bursary from the Royal Society and Nuffield Foundation. I am grateful to Professor W.J.G. Beynon for Aberystwyth facilities and for comments on this paper, and to Dr. A.D. Maude for helpful discussions. 


\section{REFERENCES}

BAmGBOYE, D.K., Investigations of north-south ionospheric movements at a low-latitude station, Radio Science, 6, 1051-1057, 1971.

Briggs, B.H. and M.G. Golley, A test for dispersion in $F$-region drifts observed by radio star scintillation method, J. Atmos. Terr. Phys., 30, 963-973, 1968.

Briggs, B.H., G.J. Phillips and D.H. Shinn, The analysis of observations on spaced receivers of fading of radio signals, Proc. Phys. Soc., B63, 106-121, 1950.

Felgate, D.G. and M.G. Golley, Ionospheric irregularities and movements observed with a large aerial array, J. Atmos. Terr. Phys., 33, 1353-1369, 1971a.

Felgate, D.G. and M.G. Golley, A cause of dispersion in ionospheric drift records, Austr. J. Phys., 24, 397-401, 1971 b.

Golley, M.G. and D.E. Rossiter, Some tests of methods of analysis of ionospheric drift records using an array of 89 aerials, J. Atmos. Terr. Phys., 32, 1215-1233, 1970.

Golley, M.G. and D.E. Rossiter, Some aspects of ionospheric drifts using partial and total reflections from the lower ionosphere, J. Atmos. Terr. Phys., 33, 701-714, 1971.

Goodman, N.R., S. KAtz, B.H. Kramer and M.T. Kuo, Frequency response from stationary noise: two case histories, Technometrics, 3, 245-268, 1961.

Gossard, E.E., Apparent movement of the spectral components in fading records of ionospherically reflected radio waves, J. Geophys. Res., 72, 1563-1569, 1967.

Haug, A. and H. Pettersen, An interpretation of asymmetric cross-correlation functions in the $D$ and $E$ region drift measurements, J. Atmos. Terr. Phys., 32, 397-403, 1970.

Hooke, W.H., Ionospheric irregularities produced by internal atmospheric gravity waves, $J$. Atmos. Terr. Phys., 30, 795-823, 1968.

Jones, D. and A.D. MAude, Evidence of wave motion in the $E$ region in the ionosphere, Nature, Lond., 206, 177-179, 1965.

Jones, D. and A.D. MAude, A new method of analysis of ionospheric drift measurements, $J$. Atmos. Terr. Phys., 30, 1487-1495, 1968.

Jones, D. and A.D. Maude, Dispersive motions in the ionosphere, J. Atmos. Terr. Phys., 34, 1241-1259, 1972.

Kent, G.S., Measurement of ionospheric movements, Rev. Geophys. Space Phys., 8, 229-288, 1970.

KENT, G.S. and R.W. WRIGHT, Movements of ionospheric irregularities and atmospheric winds, J. Atmos. Terr. Phys., 30, 657-691, 1968.

Mrtra, S.N., A radio method of measuring winds in the ionosphere, Proc. Inst. Elect. Engrs., 96, 441-446, 1949.

Papagiannis, M.D. and T.J. Elkins, Dispersive motions of ionospheric irregularities, J. Atmos. Terr. Phys., 32, 383-395, 1970.

Phillips, G.J. and M. Spencer, The effects of anisometric amplitude patterns in the measurement of ionospheric drifts, Proc. Phys. Soc., B68, 481-492, 1955. 